\title{
Concepções de validade em pesquisas qualitativas
}

Leila Giandoni Ollaik

Henrique Moraes Ziller

Universidade de Brasilia

\section{Resumo}

Este ensaio tem como objetivo expor as distintas concepções existentes sobre validade e as várias formas de aferi-la, discutindo tais questões no âmbito da pesquisa qualitativa. Como metodologia, utilizou-se a análise comparativa. Foi possível perceber que as concepções de validade podem ser agrupadas em três grandes blocos: concepções relacionadas à fase de formulação da pesquisa (validade prévia), concepções relacionadas à fase de desenvolvimento da pesquisa (validade interna) e concepções relacionadas à fase de resultados da pesquisa (validade externa). Pôde-se notar, em cada uma das fases, a presença tanto de autores com visão conceitual quantitativa, quanto de autores de perspectiva qualitativa, embora os enfoques sejam diferenciados. As concepções de validade próximas às origens positivistas preocupam-se mais com a validade na formulação e nos resultados, enquanto as concepções de validade mais interpretativistas dão maior ênfase ao desenvolvimento da pesquisa, sem desconsiderar, porém, preocupações com a formulação e com os resultados. Conclui-se que um pesquisador qualitativo pode e deve preocupar-se com a validade nas três fases da pesquisa: formulação, desenvolvimento e resultados. A validade em pesquisas qualitativas parece, assim, ser mais ampla e pormenorizada, embora menos mensurável quantitativamente.

\section{Palavras-chave}

Pesquisa qualitativa - Validade - Confiabilidade. 


\title{
Conceptions of validity in qualitative studies
}

Leila Giandoni Ollaik

Henrique Moraes Ziller

University of Brasilia

\begin{abstract}
This essay has as its objective to present the different existing conceptions on validity and on the various ways to assess it, discussing these issues within the scope of qualitative research. The methodology employs the comparative analysis. It was observed that the conceptions of validity can be grouped in three major blocks: conceptions related to the stage of formulation of the study (prior validity), conceptions related to the stage of development of the research (internal validity), and conceptions related to this stage of the results of the study (external validity). It could be noted in each of these stages the presence both of authors with a quantitative perspective and of those with a qualitative view, although their approaches are distinct. The conceptions of validity close to the positivist origins are more concerned with the validity in the formulation and in the results, whereas the more interpretivist conceptions of validity give more emphasis to the development of the research, without, however, disregarding the concern with formulation and results. It can be concluded that a qualitative researcher can and should concern him/herself with the validity of the three stages of the study: formulation, development and results. The validity in qualitative studies seems, therefore, to be wider and more detailed, albeit less quantitatively measurable.
\end{abstract}

\section{Keywords}

Qualitative research - Validity - Reliability.

Contact:

Leila G. Ollaik

leila.ollaik@gmail.com 
Este ensaio tem como objetivo expor as distintas concepções existentes sobre o conceito de validade e discuti-las no âmbito da pesquisa qualitativa. Tem como pano de fundo o debate entre as epistemologias positivista e interpretativista na construção do conhecimento.

Pesquisas qualitativas tendem a sofrer "um problema desafortunado de imagem", fazendo com "que aqueles que nunca tentaram fazer análise qualitativa propriamente dita, imaginem que se trata de uma pesquisa aleatória, sem rigor e tendenciosa". Esse "problema de imagem" também leva muitos a acharem "que fazer pesquisa qualitativa seria mais fácil do que fazer pesquisa quantitativa" (LEE; LINGS, 2008, p. 232). Nesse contexto, analisar as concepções de validade no âmbito de pesquisas qualitativas traz contribuições relevantes, permitindo uma reflexão científica que pode ajudar a desconstruir essa imagem e a instrumentalizar a pesquisa qualitativa para testes de validade.

0 ensaio está estruturado da seguinte forma: a fundamentação teórica enfoca as distintas concepções existentes de validade, apresentando, primeiramente, uma descrição de tais concepções e uma análise das várias formas possíveis de aferir validade. Em seguida, o texto discute o contexto dessas concepções e a lógica científıca subjacente a cada uma delas; finalmente, na conclusão, mediante a análise desenvolvida, essas diferentes concepções de validade em pesquisa qualitativa são agrupadas em três grandes blocos: validade prévia ou na formulação da pesquisa; validade interna ou no desenvolvimento da pesquisa; e validade externa ou de seus resultados. Tal agrupamento e a análise realizada levaram às conclusões do ensaio.

As concepções e as formas de aferição apresentadas na fundamentação teórica foram fruto de ampla pesquisa sobre métodos qualitativos, incluindo análise de artigos sobre validade publicados em cinco periódicos internacionais especializados em pesquisas qualitativas, quais sejam: Qualitative Research Journal; The Qualitative Report; Quality \& Quantity
- International Journal of Methodology; International Journal of Qualitative Methods; e Qualitative Inquiry. Buscou-se identificar, no período de 2001 a 2010, primeira década do século XXI, artigos que contivessem o termo validade no título. Dentre estes, foram utilizados os textos que trataram de forma mais abrangente a questão da validade em pesquisas qualitativas. As conclusões apresentam sugestão para pesquisas futuras.

\section{Concepções de validade}

A fundamentação teórica aqui disposta traz distintas concepções de validade, apresentando uma exposição de diversas formas de aferir a validade, com ênfase em pesquisas qualitativas, e uma breve discussão sobre o contexto e a lógica subjacentes a cada concepção e a cada tipo de pesquisa científica. Ao final da seção, tais elementos serão relacionados.

Dicionários definem validade como qualidade - ou condição - de válido. 0 termo válido, por sua vez, é definido como aquilo que tem valor, que é valioso; aquilo que tem saúde, que é sadio, são; aquilo que é vigoroso; aquilo que surte efeito, que é eficaz (FERREIRA, 2009). 0 mesmo termo é também definido como aquilo que contém premissas das quais a conclusão pode ser logicamente extraída (SACCONI, 2010).

Em pesquisa científica, a validade assume concepções mais complexas. A elaboração original do conceito vem de métodos quantitativos nos quais a validade é a extensão em que uma medida representa corretamente o conceito do estudo, ou seja, o grau em que a medida está livre de qualquer erro. A validade seria referente à semelhança entre o conceito e suas medidas, ao grau em que uma medida representa precisamente o que se espera. A garantia da validade começaria com a compreensão direta do que deve ser medido, sendo, portanto, uma questão prioritariamente de formulação da pesquisa (HAIR Jr. et al., 2009).

Em pesquisas quantitativas, como o pano de fundo é a filosofia positivista, a validade é 
um atributo que se relaciona com a objetividade, com a possibilidade de repetição do experimento, com o fato de a pesquisa estar aberta à verificação por outras pessoas e com a capacidade de generalização. Assim, para que uma pesquisa quantitativa seja considerada válida, outro pesquisador deve poder desenvolvê-la e chegar ao mesmo resultado. Nessa concepção, a ciência é vista com objetividade e tem caráter explicativo (MARTINS, 2004).

Os critérios tradicionais adotados para verificar a existência de validade em pesquisas têm suas raizes nessa tradição positivista. De certa forma, o próprio positivismo tem sido definido por uma teoria sistemática de validade (GOLAFSHANI, 2003).

A validade em pesquisa qualitativa é um tema explorado já há cerca de meio século, com maior ênfase em anos recentes. Embora sua origem sejam os pressupostos adotados na pesquisa quantitativa, ela vem sendo adaptada e utilizada também na pesquisa qualitativa (CHO; TRENT, 2006).

Em pesquisas qualitativas, a concepção de validade assume formas distintas, pois a discussão sobre escalas de medição não se aplica a métodos qualitativos, sendo necessária a compreensão da validade em outra perspectiva. Para além de questões de formulação, naquilo que pode ser compreendido como validade prévia, a validade busca indicar o que constitui uma pesquisa bem feita, confiável, merecedora de ser tornada pública para contribuir para o conhecimento, ou - conforme a definição apresentada inicialmente para o termo válido - que tem valor, eficaz. Nesse sentido, adaptando o conceito quantitativista para pesquisas qualitativas, verificar a validade de uma pesquisa seria determinar se ela de fato mede verdadeiramente o que o pesquisador propôs-se a medir, se seus processos metodológicos são coerentes e se seus resultados são consistentes.

A validade pode ser vista genericamente como a correspondência entre a pesquisa e a realidade (BIANCHI; IKEDA, 2008). Cabe questionar, no entanto, se é possível fazer essa trans- posição de maneira a encontrar uma concepção de validade que atenda tanto à pesquisa quantitativa como à pesquisa qualitativa. Dentro do universo de pesquisas qualitativas, há várias possibilidades de definições e critérios de validade. É preciso conhecer com clareza o contexto no qual se está atuando para defınir com coerência a concepção de validade que será adotada e explicitar os critérios que então serão úteis. Daí decorre que, em pesquisa qualitativa, a concepção de validade e seu método de aferição são definidos de diversas formas. Não se trata de concepções únicas, fixas ou universais, mas de um construto fortemente ligado aos processos e às intenções de cada projeto e de cada metodologia de pesquisa (GOLAFSHANI, 2003). Diferentes contextos podem propiciar diferentes adaptações dos métodos utilizados.

Nas mais diversas concepções adotadas, porém, a validade refere-se à verificação dos resultados como verdadeiros e confiáveis. Ela estaria relacionada ao fato de os resultados refletirem com precisão a situação analisada e serem confiáveis, no sentido de que não haveria razões para deles duvidar; ou seja, a pesquisa é válida se as evidências fornecem o apoio necessário às suas conclusões (GUION, 2002). Nesse particular, mais uma vez, denota-se a influência positivista que compreende a essência da pesquisa como a busca da verdade.

No entanto, a pesquisa qualitativa busca descrever e compreender um fenômeno, e não explicá-lo ou fazer previsões. Tais descrição e compreensão estão restritas a um contexto específico a partir do qual se chega a um tipo de conhecimento distinto do que é alcançável por procedimentos estatísticos ou por outras formas de quantificação. Em vez de explicar, busca-se descrever. Em vez de prever, busca-se compreender. Em vez de generalizar, busca-se a possibilidade de extrapolação para situações com contextos similares (GOLAFSHANI, 2003).

Uma das principais críticas feitas à pesquisa qualitativa é que a ela falta representatividade, não permitindo generalizações e sendo por demais subjetiva, aspecto este decorrente 
principalmente da proximidade entre pesquisador e pesquisado. Além disso, ela sofre críticas por ter caráter descritivo e narrativo, e não explicativo. Tais críticas, entretanto, só seriam válidas a partir do paradigma quantitativo, visto que, em pesquisas qualitativas, a intenção não é generalizar, mas sim descrever, analisar, buscar compreender. Nessa perspectiva, a validade estaria relacionada com a coerência interna da pesquisa (MARTINS, 2004).

Ou seja, há concepções que dão mais ênfase à validade dos resultados, também denominada validade externa, e há concepções que dão mais ênfase à validade do processo, do método, também denominada validade interna.

Para Mirka Koro-Ljungberg (2010), a validade em pesquisas qualitativas está mais fortemente relacionada com a responsabilidade no tratamento das informações obtidas e nas decisões do pesquisador, envolvendo intensa preocupação ética. Nessa linha, de validade interna, de processo, há também a concepção de validade transacional. Esta age em uma perspectiva micro, na qual o cerne da questão está relacionado com a interação entre o pesquisador, o pesquisado e os dados encontrados, de maneira a alcançar a maior identidade possível entre sentimentos, experiências, valores e opiniões coletados, e a narrativa feita pelo pesquisador sobre eles (CHO; TRENT, 2006).

A validade transacional admite a utilização de diversos métodos já conhecidos, e compõe, junto com a validade transformacional, duas grandes linhas de validação. Nesse segundo grupo, o que está em questão não é mais a verificação da validade de procedimentos, mas, para além disso, trata-se de uma abordagem na qual a validação equivale ao impacto causado pela realização da pesquisa. Mediante o esforço de pesquisa, haveria um resultado de emancipação em direção à mudança social (CHO; TRENT, 2006).

A validade transacional se caracterizaria por seu foco procedimental, mais especificamente, na relação entre pesquisador e pesquisado, enquanto a validade transformacional se caracterizaria pelo processo, no qual os procedimentos perdem relevância em favor do impacto causado pela pesquisa em si mesma (CHO; TRENT, 2006).

Jeasik Cho e Allen Trent (2006) concluem seu trabalho com a apresentação de um modelo que prevê uma variada combinação de métodos de validação em função de distintos objetivos que uma pesquisa qualitativa pode ter. A partir dos objetivos de cada pesquisa, derivam-se concepções de validade mais aderentes a eles. Para os autores, os objetivos da pesquisa qualitativa podem ser: busca da verdade, descrição densa, desenvolvimentista, ensaio pessoal e praxis/social.

No ensaio praxis/social, a verificação da validade assemelha-se à da validade transformacional, com a diferença de que não se trata mais de impacto advindo do esforço da pesquisa, mas da interação entre pesquisador e participantes, e de sua posterior atitude quotidiana transformada pelo processo de pesquisa (CHO; TRENT 2006).

Tal proposição de adotar métodos de validação conforme o objetivo de cada pesquisa consiste em uma perspectiva integradora e não excludente. Com ela, almeja-se alcançar uma proposta holística de validade, a salvo de técnicas mágicas, livrando as pesquisas de caminhos e resultados equivocados (CHO; TRENT, 2006).

Apesar dessas diferenças, tanto pesquisas quantitativas quanto pesquisas qualitativas, independentemente da argumentação que se faça a respeito da validade de uma pesquisa qualitativa, procuram demonstrar que seus estudos são críveis, confiáveis e válidos.

\section{Como aferir validade}

Há diferentes técnicas para aferição da validade de uma pesquisa científıca, conforme a concepção de validade que esteja sendo utilizada. A validade em pesquisa qualitativa tem sido operacionalizada de várias maneiras, e todas as concepções apresentam algum nível de apropriação adequada aos diversos desenhos desse tipo de pesquisa (ONWUEGBUZIE; LEECH, 2007). 
Para verificar a validade externa de uma pesquisa qualitativa, a opção por adotar, sem adaptações, a concepção positivista, verificando a capacidade de replicação e generalização dos resultados, redundaria em um esforço com poucas perspectivas de sucesso (ROCHA-PINTO; FREITAS; MAISONNAVE, 2008). Daí se conclui que, quando se trata de pesquisas qualitativas, possivelmente se dá maior relevância à validade interna, visto que a generalização não é, usualmente, objetivo da pesquisa qualitativa.

As técnicas no âmbito da validade transacional são um meio para assegurar a maior aderência possivel entre os dados coletados e interpretados e a realidade; incluem abordagens para aferição da validade, como a triangulação. Mediante a utilização desses métodos, os informantes da pesquisa são instados a confirmar se a interpretação do pesquisador corresponde à sua realidade, pois a construção que o pesquisador faz da realidade será, inevitavelmente, uma reconstrução, uma interpretação (CHO; TRENT, 2006).

Em relação à concepção interpretativista, Hartmut Günther (2006) apresenta uma lista de diversas perguntas que orientariam a avaliação da validade interna de pesquisas qualitativas, tais como: As perguntas da pesquisa estão claramente formuladas? 0 delineamento da pesquisa é consistente com seu objetivo e com suas perguntas? Os paradigmas e os construtos analíticos foram bem explicitados? A posição teórica e as expectativas do pesquisador foram evidenciadas? Adotaram-se regras explícitas nos procedimentos metodológicos e analíticos? Os procedimentos metodológicos e analíticos estão bem documentados? Os dados foram coletados em todos os contextos, tempos e pessoas sugeridos pelo delineamento? 0 detalhamento da análise leva em conta resultados não esperados e contrários ao esperado? A discussão dos resultados leva em conta possíveis alternativas de interpretação? Os resultados são congruentes com as expectativas teóricas? Explicitou-se a teoria que pode ser derivada dos dados e utilizada em outros contextos? Os resultados são acessíveis para a comunidade acadêmica e para os usuários no campo? Os resultados estimulam ações - básicas e aplicadas - futuras?

Segundo Günther (2006), tal método é consistente com os princípios tanto da pesquisa qualitativa, quanto da pesquisa quantitativa, pois os critérios ofereceriam o nível de validação numa gradação qualitativa, e não mediante um valor numérico.

0 método de triangulação é frequentemente utilizado para demonstrar validade em pesquisas científicas. A origem do termo triangulação está nas ciências que se utilizam de pesquisas sobre terra e sobre navegação, referindo-se a um método simples para determinar a posição de um ponto mediante observação de dois outros pontos adicionais (FARMER et al., 2006). Migrando das ciências exatas, a triangulação foi adotada em pesquisas na área de saúde e em pesquisas sociais por psicólogos quantitativos. Depois, passou a ser utilizada em várias áreas, por se acreditar que ela permite uma compreensão multidimensional de problemas complexos. Seu objetivo principal é aumentar a validade da pesquisa, garantindo que os resultados e suas interpretações sejam confráveis (LINCOLN; GUBA, 2006).

A triangulação implica a utilização de abordagens múltiplas a fim de evitar distorções devido a um método, uma teoria ou um pesquisador (GÜNTHER, 2006). Ela visa controlar vieses e enriquecer constatações, bem como confirmar e reafirmar validade e confiabilidade. Para Tracy Farmer et al. (2006), triangulação é um enfoque metodológico que contribui para a validade dos resultados de uma pesquisa quando são utilizados múltiplos métodos, teorias, fontes e pesquisadores.

Lisa Guion (2002) define cinco tipos de triangulação. 0 primeiro é a triangulação de dados, em que se utilizam diferentes fontes de dados ou de informações para se chegar ao mesmo resultado; é o tipo mais conhecido e o de mais fácil implementação. 0 segundo tipo é a triangulação de pesquisadores, no qual diferentes pesquisadores estudam o 
mesmo tema utilizando-se das mesmas técnicas (entrevista, observação, estudo de caso, grupos focais). Se os diferentes pesquisadores chegarem às mesmas conclusões, então fica estabelecida a validade da pesquisa. 0 terceiro tipo é a triangulação de teorias, em que profissionais de campos de estudo distintos como economia, antropologia, ciência política, administração - envolvem-se na pesquisa, a partir de diferentes perspectivas, para interpretar o mesmo conjunto de informações. 0 quarto tipo, também popular e amplamente utilizado, é a triangulação metodológica, que utiliza métodos tanto qualitativos quanto quantitativos para estudar um problema. Alguns autores, como José Luis Neves (1996), chamam de triangulação apenas esse último tipo, qual seja, a combinação de métodos quantitativos e qualitativos em uma mesma pesquisa. Finalmente, o quinto método é a triangulação ambiental, que envolve o uso de diferentes locais ou diferentes fatores-chave para a pesquisa em questão, como a hora do dia, o dia da semana, ou a estação do ano. Há que se identificar um fator ambiental que seja relevante e que possa influenciar a informação; caso se chegue à mesma conclusão modificando o fator ambiental, está estabelecida a validade da pesquisa (GUION, 2002). Paulien Meijer, Nico Verloop e Douwe Beijard (2002) também identificam esses cinco tipos de triangulação, com algumas diferenças, e os apresentam em ordem distinta.

Há, ainda, a validação da pesquisa pelos pares, pela comunidade acadêmica, tal como mencionado por Alda Judith Alves-Mazzotti (2006) ao tratar da temática de estudo de caso. Não se trata, nessa situação, da triangulação de pesquisadores. Antes, a autora concebe um diálogo do pesquisador com a comunidade acadêmica, o que confere relevância e confiabilidade à pesquisa, num processo de produção coletiva de conhecimento.

Para verificação da confiabilidade quando se trabalha com grupos focais, é possível verificar se o processo da pesquisa foi bem or- ganizado, planejado e baseado em um contrato ético de participação assumido por todos os elementos do grupo (RESSEL et al., 2008).

Pode-se também manter um protocolo para verificar confiabilidade e garantir validade em pesquisas qualitativas, e existem mais de 24 métodos com essa finalidade. Alguns desses métodos são: envolvimento prolongado; observação persistente; triangulação; registro de tudo; checagem com os membros; análise das evidências; verificação por representatividade; explicitação do viés do pesquisador; comparações; experimentação teórica (ir aonde os dados levarem, e não manipulá-los); verificação dos significados de casos extremos; utilização dos casos extremos; eliminação de relações espúrias; análise das surpresas encontradas nos dados; elaboração de relações; consulta a um colega para que este aja como advogado do diabo; entre outros (ONWUEGBUZIE; LEECH, 2007).

Ademais, pode-se verificar a qualidade de uma pesquisa qualitativa por sua transparência, sua coerência e sua comunicabilidade, as quais construiriam a justificativa das interpretações. Por transparência entende-se que o leitor da pesquisa é capaz de identificar com clareza os processos que foram utilizados na coleta e na análise dos dados; por coerência entende-se que os construtos teóricos estão ajustados e coerentes com a análise desenvolvida; por comunicabilidade entende-se que a pesquisa faz sentido para os leitores, para o pesquisador e para os pesquisados (ROCHA-PINTO; FREITAS; MAISONNAVE, 2008).

Em suma, há várias estratégias para estabelecer validade em pesquisas qualitativas (MORSE et al., 2002). Há também numerosos métodos e propostas de conciliação entre eles, bem como análises variadas sobre seus usos em pesquisas qualitativas. Há, enfim, ampla reflexão sobre a utilização da concepção e da aferição da validação em pesquisas qualitativas, embora a escolha do pesquisador dependa fundamentalmente do contexto e da lógica científica subjacente a cada concepção. 


\section{Contexto das concepções e lógica científica subjacente}

A pesquisa no âmbito das ciências naturais parte do pressuposto de que é possível identificar uma realidade preexistente, de maneira objetiva e sem a influência do pesquisador, a partir de métodos neutros que vão permitir a identifıcação da existência de leis que regem o funcionamento do universo e controlam toda a existência, em última análise. A validação desse tipo de pesquisa utiliza-se, necessariamente, de instrumentos que permitem comparar a realidade dos fenômenos observados com suas medidas e as representações dessas medidas (MARTINS, 2004).

A pesquisa no âmbito das ciências sociais identificou outra possibilidade e, em grande medida, valeu-se dela; é a identificação da realidade construída, em que o pesquisador interage com o objeto da pesquisa em um processo no qual sua própria cognição influi no resultado. Trata-se, portanto, da construção do conhecimento a partir de uma postura interpretativa, o que implica a possibilidade de que pesquisa e pesquisador sejam participantes ativos do processo de construção do conhecimento, e não mais lhes impõe uma postura neutra, afastada, como requer o positivismo (GOLAFSHANI, 2003).

De acordo com os positivistas, qualquer pesquisa científıca, por definição e também por suas características intrínsecas, deve necessariamente ser quantitativa. Ou seja, nessa concepção, só são consideradas científicas as pesquisas baseadas em dados e que utilizam instrumentos de mensuração. Eles acreditam que os métodos qualitativos não dão origem a dados confiáveis. Por sua vez, os interpretativistas desconfiam dos positivistas afirmando que estes não realizam pesquisas válidas, porque não se colocam no lugar do objeto estudado, do sujeito (ROCHA-PINTO; FREITAS; MAISONNAVE, 2008).

0 conflito existente entre pesquisa quantitativa e qualitativa é manifesto e envolve até mesmo esferas estatais. Cho e Trent (2006) narram os esforços do governo norte-americano para impor conceitos quantitativos como base do paradigma a ser seguido na construção do conhecimento científico.

Pesquisadores qualitativistas consideram que a concepção de validade definida em termos quantitativos é inadequada, pois estes podem não ser aplicáveis ao paradigma da pesquisa qualitativa. Nessa linha, foram desenvolvidos outros conceitos que estão para a pesquisa qualitativa assim como a validade está para pesquisa quantitativa, por exemplo, fidedignidade, rigor e qualidade (GOLAFSHANI, 2003).

Cho e Trent (2006) demonstram, com grande sutileza, a natureza do problema ao tratarem do conceito de validade transacional:

Em grande medida, esse enfoque assume que a pesquisa qualitativa pode ser mais confiável na medida em que algumas técnicas, métodos e/ou estratégias sejam empregadas durante o desenvolvimento da pesquisa. Em outras palavras, técnicas são vistas como uma forma de garantir reflexão precisa da realidade (ou, ao menos, da construção da realidade pelos participantes da pesquisa). (p. 322)

A ideia de que a adoção de determinadas medidas vá conceder maior credibilidade à pesquisa qualitativa parece trazer em si a noção subjacente de que ainda lhe falta um quantum de credibilidade, mesmo que tal medida seja indefınida. A solução seria, então, a adoção de técnicas, métodos e estratégias a serem introduzidos no processo de pesquisa, de maneira a se obter um acurado reflexo da realidade. Dado que a obtenção de um acurado reflexo da realidade decorre de uma categoria positivista, Cho e Trent (2006) fazem a ressalva de que pode se tratar, na verdade, de uma construção da realidade feita pelo participante da pesquisa, alcançando a perspectiva antipositivista da construção do conhecimento. 
Cho e Trent (2006) fazem, ainda, referência a uma mudança conceitual que eles observaram nos trabalhos de Yvonna Lincoln e Egon Guba de 1985, 1989 e 2000. No trabalho de 1985, os autores referiam-se com naturalidade à triangulação como método de aferição da acurácia de dados em pesquisa qualitativa. Já em 1989, o trabalho havia evoluído para uma reflexão acerca das implicações positivistas do método da triangulação. Posteriormente, no texto de 2000, vê-se que Lincoln e Guba consideram duas perspectivas de validade: a perspectiva positivista, em que a validade é absolutamente alcançável, e a perspectiva construtivista, na qual a validade nunca pode ser alcançada, mas, ao contrário, tem de ser checada indefinidamente.

Entre outras conclusões, Cho e Trent (2006) afırmam, afinal, que é equivocado o objetivo de alguns cientistas em definir métodos e estratégias de validação da pesquisa qualitativa a partir das estratégias convencionais da pesquisa quantitativa. Também Nahid Golafshani (2003) entende que conceitos como validade e triangulação, caso intencionem alcançar o status de conceitos relevantes em pesquisa qualitativa, devem ser redefinidos a partir de um ponto de vista qualitativo.

De fato, a utilização da triangulação como instrumento de validação em pesquisas quantitativas e qualitativas deve considerar a distinção entre os universos positivista e interpretativista. Enquanto, na pesquisa quantitativa, qualquer exceção pode levar à não confirmação da hipótese, na pesquisa qualitativa, exceções podem levar à modificação ou até à construção de teorias (GOLAFSHANI, 2003).

Cho e Trent (2006), quando propõem o enfoque de distintos conceitos de validade para distintos objetivos de pesquisa, acabam impondo a relativização da busca e do conhecimento da verdade como propósitos finais da pesquisa científıca, valores tão caros ao positivismo. Eles fazem isso ao indicarem cinco tipos de propósitos distintos na pesquisa qualitativa, dentre os quais apenas um refere-se à busca da ver- dade, sendo que outro aponta, inclusive, para um novo referencial em relação ao resultado de uma pesquisa: o objetivo praxis/social, que compreende valores como emancipação cidadã e justiça social.

As diversas definições de validade em pesquisa qualitativa, assim como a diversidade dos métodos de validação existentes, manifestam a distinção desse conceito em relação àquele desenvolvido no âmbito das pesquisas quantitativas, o qual é menos fluido, mais concreto e facilmente apreensível.

\section{Conclusões}

A partir das distintas concepções, formas de aferição e lógicas subjacentes, desenvolveu-se uma análise agrupando as diversas concepções de validade em pesquisa qualitativa. Foi possível perceber que tais concepções podem ser agrupadas em três grandes blocos: concepções mais relacionadas à fase da formulação da pesquisa (validade prévia), concepções mais relacionadas à fase de desenvolvimento da pesquisa (validade interna) e concepções mais relacionadas à fase de resultados da pesquisa (validade externa).

0 agrupamento foi inspirado no texto de David Brinberg (1982), em sua concepção básica acerca de três momentos distintos para aferição da validade em pesquisas qualitativas: a formulação, o desenvolvimento e o resultado. A partir daquilo que foi pesquisado e apresentado ao longo deste ensaio, foram adicionados outros elementos ao agrupamento, o qual é apresentado no Quadro 1.

Nota-se a presença, em cada uma das três fases, tanto de autores com visão conceitual quantitativa, quanto de autores de perspectiva qualitativa, embora os enfoques sejam diferenciados. Na formulação, pode-se ter uma validade quantitativa sobre se a medida representa precisamente a realidade, ou uma validade qualitativa, que é construída pelo pesquisador e pode ser definida de diversas formas. No desenvolvimento, um enfoque mais próximo às 
Quadro 1 - Diferentes concepções de validade em pesquisa qualitativa, agrupadas em três grandes blocos

\begin{tabular}{|c|c|c|c|}
\hline $\begin{array}{l}\text { Concepções de } \\
\text { validade }\end{array}$ & Especificações & Tipos e características & Principais autores \\
\hline \multirow{5}{*}{ 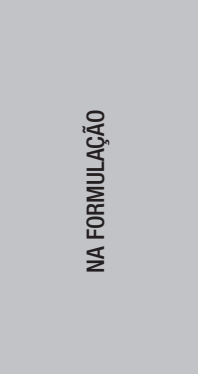 } & Validade prévia & $\begin{array}{l}\text { Correspondência entre a pesquisa e a } \\
\text { realidade }\end{array}$ & BIANCHI; IKEDA, 2008 \\
\hline & $\begin{array}{l}\text { Grau em que uma medida representa } \\
\text { exatamente } 0 \text { que se espera }\end{array}$ & Convergente, discriminante e monológica & \multirow{3}{*}{ HAIR Jr. et al., 2009} \\
\hline & Compreensão do que deve ser medido & & \\
\hline & $\begin{array}{l}\text { Possibilidade de repetir o experimento, } \\
\text { replicabilidade }\end{array}$ & & \\
\hline & Construto fluido & Construída pelo pesquisador & GOLAFSHANI, 2003 \\
\hline \multirow{3}{*}{ 일 } & Validade interna & $\begin{array}{l}\text { Contém premissas das quais a conclusão } \\
\text { pode ser logicamente extraída }\end{array}$ & SACCONI, 2010 \\
\hline & $\begin{array}{l}\text { Foco nos procedimentos, ênfase na } \\
\text { metodologia }\end{array}$ & Transparência, responsabilidade e ética & $\begin{array}{l}\text { KORO-LJUNGBERG, } \\
2010\end{array}$ \\
\hline & Transacional & Interação entre pesquisador e pesquisado & CHO; TRENT, 2006 \\
\hline \multirow{5}{*}{ 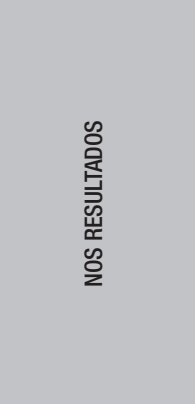 } & \multirow[t]{2}{*}{ Validade externa } & $\begin{array}{l}\text { Capacidade de generalização } \\
\text { ou extrapolação dos resultados } \\
\text { (replicabilidade) }\end{array}$ & HAIR Jr. et al., 2009 \\
\hline & & $\begin{array}{l}\text { Verificar se os resultados são verdadeiros } \\
\text { e confiáveis }\end{array}$ & GUION, 2002 \\
\hline & Impacto da pesquisa & Que surte efeito, eficaz & FERREIRA, 2009 \\
\hline & Transformacional & Emancipação social & \multirow{2}{*}{ CHO; TRENT, 2006} \\
\hline & & $\begin{array}{l}\text { Descrever, compreender e extrapolar para } \\
\text { situações }\end{array}$ & \\
\hline
\end{tabular}

origens positivistas seria o da lógica, embora predomine uma perspectiva mais qualitativa relacionada a ética, interação, comportamento, responsabilidade e coerência. Nos resultados, observa-se tanto a validade quantitativa como poder de generalizações e predições, quanto a validade qualitativa como poder de compreensão e transformação.

Tal agrupamento mostrou, entre outras coisas, que as concepções de validade próximas às origens positivistas preocupam-se mais com a validade na formulação e nos resultados; por sua vez, as concepções de validade mais interpretativistas dão maior ênfase ao desenvolvimento da pesquisa, sem desconsiderar, porém, preocupações com a formulação e com os re- sultados. A validade em pesquisas qualitativas parece ser, assim, mais ampla e pormenorizada, embora menos mensurável.

Vemos, conforme o Quadro 1, que diversos conceitos de validade utilizados em pesquisas qualitativas foram herdados de pesquisas quantitativas. Essa incorporação exigiu adaptações, uma vez que os contextos de atuação e as epistemologias subjacentes a cada tipo de pesquisa são distintos.

Mesmo autores como Cho e Trent (2006), que trabalham a questão das transições epistemológicas dos métodos de validação, também adotam proposições que inserem mecanismos originalmente positivistas nos métodos de validação qualitativos. 
A obrigatoriedade de que a construção do pesquisador não seja uma reconstrução e de que as informações obtidas sejam checadas, inclusive com a imposição de que percepções pessoais dos pesquisadores sejam excluídas da pesquisa, é um comportamento esperado no âmbito da pesquisa em ciências naturais. No entanto, no que concerne à pesquisa qualitativa, esse condicionamento precisa ser adaptado, pois acaba por propor o tipo de distanciamento esperado entre pesquisador e a realidade pesquisada na tradição positivista.

Uma adaptação condizente com a lógica qualitativa é o modelo de validade transformacional, por possibilitar que, ao lado da busca pela verdade, seja incluído um objetivo de pesquisa mais alinhado com a tradição interpretativa, pois se aproxima de valores como justiça social e ética (CHO; TRENT, 2006).

Em decorrência desta análise, vê-se que um pesquisador pode e deve preocupar-se com a validade nas três fases da pesquisa qualitativa: formulação, desenvolvimento e resultados. A forma que essa preocupação irá assumir va- ria de pesquisador para pesquisador, conforme suas orientações filosóficas, epistemológicas e científicas, sendo de fundamental importância, no entanto, manter coerência ao longo de toda a pesquisa.

Em suma, há várias concepções de validade; tal conceito surge no âmbito de pesquisas quantitativas e é adaptado para pesquisas qualitativas. A adaptação vem ocorrendo de forma ampla e pode ser notada nos três grandes blocos de validade: a prévia (na formulação da pesquisa), a interna (no desenvolvimento da pesquisa) e a externa (nos resultados da pesquisa). Há diversas concepções e diferentes técnicas para aferição de validade, e o pesquisador qualitativo deve avaliar a escolha por métodos de validação a fim de manter a coerência entre epistemologia, métodos e técnicas no decorrer de toda a pesquisa.

Sugere-se investigar acerca de justiça social e ética como valores subjacentes aos objetivos de pesquisa, o que talvez possa sinalizar um novo e mais adequado caminho para a validade em pesquisas com epistemologia interpretativa. 


\section{Referências}

ALVES-MAZZOTTI, Alda Judith. Usos e abusos dos estudos de caso. Cadernos de Pesquisa, v. 36, n. 129, p. 637-651, set./dez. 2006. BIANCH, Eliane Maria P. Giavina; IKEDA, Ana Akemi. Usos e aplicações da grounded theory em Administração. GESTÃo.Org, v. 6, n. 2, p. 231-248, 2008.

BRINBERG, David. Validity concepts in research: an integrative approach. Advances in Consumer Research, v. 9, p. 40-44, 1982. CHO, Jeasik; TRENT, Allen. Validity in qualitative research revisited. Qualitative Research Journal, v. 6, n. 3, p. 319-340, 2006.

FARMER, Tracy et al. Developing and implementing a triangulation protocol for qualitative health research. Qualitative Health Research, v. 16, p. 337-394, 2006.

FERREIRA, Aurélio B. de Holanda. Novo Dicionário Aurélio da Língua Portuguesa. 4. ed. Curitiba: Positivo, 2009.

GOLAFSHANI, Nahid. Understanding reliability and validity in qualitative research. The Qualitative Report, v. 8, n. 4, p. 597-607, dec. 2003.

GÜNTHER, Hartmut. Pesquisa qualitativa versus pesquisa quantitativa: esta é a questão? Psicologia: Teoria e Pesquisa, Brasília, v. 22, n. 2, p. 201-210, maio/ago. 2006.

GUION, Lisa A. Triangulation: establishing the validity of qualitative studies. Gainesville: University of Florida, 2002.

HAIR Jr., Joseph F. et al. Análise multivariada de dados. 6. ed. Porto Alegre: Bookman, 2009.

KORO-LJUNGBERG, Mirka. Validity, responsibility, and aporia. Qualitative Inquiry, v. 16, n. 8, p. 603-610, 2010.

LEE, Nick; LINGS, lan. Doing business research: a guide to theory and practice. Londres: Sage Publications Ltd., 2008.

LINCOLN, Yvonna S.; GUBA, Egon G. Controvérsias paradigmáticas, contradições e confluências emergentes. In: DENZIN, Norman K.; LINCOLN, Yvonna S. 0 planejamento da pesquisa qualitativa: teorias e abordagens. 2. ed. Porto Alegre: Artmed, 2006. p. 169-192.

MARTINS, Heloísa Helena T. de Souza. Metodologia qualitativa de pesquisa. Educação e Pesquisa, São Paulo, v. 30, n. 2, p. 289300, maio/ago. 2004.

MEIJER, Paulien C.; VERLOOP, Nico; BEIJAARD, Douwe. Multi-method triangulation in a qualitative study on teachers' practical knowledge: an attempt to increase internal validity. Quality \& Quantity: International Journal of Methodology, v. 36, n. 2, p. 145$167,2002$.

MORSE, Janice M. et al. Verification strategies for establishing reliability and validity in qualitative research. International Journal of Qualitative Methods, v. 1, n. 2, p. 13-22, 2002.

NEVES, José Luis. Pesquisa qualitativa: características, usos e possibilidades. Caderno de Pesquisas em Administração, São Paulo, v. 1, n. 3, $2^{\circ}$ sem., 1996.

ONWUEGBUZIE, Anthony J.; LEECH, Nancy L. Validity and qualitative research: an oxymoron? Quality \& Quantity: International Journal of Methodology, v. 41, n. 2, p. 233-249, 2007.

RESSEL, Lúcia Beatriz et al. 0 uso do grupo focal em pesquisa qualitativa. Texto \& Contexto - Enfermagem, Florianópolis, v. 17, n. 4, p. 779-786, out./dez. 2008. 
ROCHA-PINTO, Sandra Regina; FREITAS, Angilberto Sabino; MAISONNAVE, Paulo Roberto. Métodos interpretativistas em Administração: as implicações para o(a) pesquisador(a). In: ENCONTRO DA ASSOCIAÇÃO NACIONAL DE PÓS-GRADUAÇÃO E PESQUISA EM ADMINISTRAÇÃO, 32., 2008, Rio de Janeiro. Anais... Rio de Janeiro: ANPAD, 2008.

SACCONI, Luiz Antonio. Grande Dicionário Sacconi da Língua Portuguesa. São Paulo: Nova Geração, 2010.

Recebido em: 08.04.2011

Aprovado em: 25.08.2011

Leila G. Ollaik é mestre em Políticas Públicas pela Oxford University, doutoranda em Administração, mestre em Ciência Política e bacharel em Economia pela Universidade de Brasília. Especialista em Políticas Públicas e Gestão Governamental do Gov. Federal.

Henrique M. Ziller é mestrando em Administração Pública na Universidade de Brasília e bacharel em Comunicação Social pelo Centro Universitário de Brasília. Auditor do Tribunal de Contas da União. Voluntário no Instituto de Fiscalização e Controle. E-mail: henrique@ziller.com.br. 
